\title{
Squares in products from a block of consecutive integers
}

\author{
by
}

\author{
R. Balasubramanian (Madras) and T. N. Shorey (Bombay)
}

1. Let $k \geq t \geq 2, m \geq 0, y \geq 1$ be integers and write $d_{1}, \ldots, d_{t}$ for distinct positive integers not exceeding $k$. The letter $b$ denotes a positive integer such that the greatest prime factor of $b$ is less than or equal to $k$. We put

$$
F(k)=k(\log k) /(\log \log k) \quad \text { for } k \geq 3 .
$$

For a real number $\theta$ and $k \geq 27$, we define

$$
\mu_{k}(\theta)=k\left(1-\frac{\log \log k}{\log k}+\frac{\log \log \log k}{\log k}+\frac{\theta}{\log k}\right) .
$$

Finally, we recall that $\gamma$ is Euler's constant.

We consider the equation

$$
\left(m+d_{1}\right) \ldots\left(m+d_{t}\right)=b y^{2} .
$$

It follows from a theorem of Baker [1] that equation (1) with $t \geq 3$ implies that $\max (b, m, y)$ is bounded by an effectively computable number depending only on $k$. Erdös [2] and Rigge [8], independently, proved in 1939 that equation (1) with $t=k$ and $b=1$ is not possible. Thus, the product of two or more consecutive positive integers is never a square. In fact, Erdős [3, p. 88] observed in 1955 that his method allows to show that there exists an absolute constant $C_{1}>0$ such that equation (1) with $b=1$ and

$$
m>k^{2}, \quad t \geq k-C_{1} k /(\log k)
$$

implies that $k$ is bounded by an effectively computable absolute constant. Further, Erdős [3, p. 88] stated in 1955 that he had no proof of the following sharpening of the preceding result:

Let $\varepsilon>0$. The equation (1) with $b=1$ and

$$
m>k^{2}, \quad t \geq k-(1-\varepsilon) k \frac{\log \log k}{\log k}
$$

implies that $k$ is bounded by an effectively computable number depending only on $\varepsilon$. 
Shorey [9] applied in 1986 Brun's sieve and an estimate of Sprindžuk [12] on the magnitude of integral solutions of a hyperelliptic equation to prove that equation (1) with

$$
m>k^{2}, \quad t \geq k-(1-\varepsilon) k \frac{\log \log \log k}{\log k}
$$

implies that $k$ is bounded by an effectively computable number depending only on $\varepsilon$. Further, Shorey [10] relaxed in 1987 the assumption (3) to (2). In this paper, we combine the arguments for the proofs of the preceding results of Shorey to obtain a further relaxation of the assumption (2).

TheOrem 1. Let $k \geq 27$. There exist effectively computable absolute constants $\theta_{0}$ and $C_{2}$ such that equation (1) with

$$
m>k^{2}
$$

and

$$
t \geq \mu_{k}\left(\theta_{0}\right)
$$

implies that

$$
k \leq C_{2} .
$$

Since $\mu_{k}(\theta)$ is an increasing function of $\theta$, we observe that the assumption (5) can be replaced by $t \geq \mu_{k}(\theta)$ for any $\theta>\theta_{0}$. For an integer $\nu>1$, we define $P(\nu)$ to be the greatest prime factor of $\nu$ and we write $P(1)=1$. If equation (1) with $P(y)>k$ is valid, we can find an integer $i$ with $1 \leq i \leq k$ such that $m+d_{i} \geq(k+1)^{2}$, which implies that $m>k^{2}$. Consequently, we observe that the assumptions (4) and (5) in Theorem 1 can be replaced by

$$
P(y)>k, \quad t \geq \mu_{k}\left(\theta_{0}\right) .
$$

If $P(y) \leq k$, we observe from (1) that

$$
P\left(m+d_{i}\right) \leq k \text { for } 1 \leq i \leq t,
$$

which implies that

$$
t \leq \frac{k \log k}{\log m}+\pi(k),
$$

by a well-known argument of Erdős [3, Lemma 3]; see also [4, Lemma 2.1]. In (6), we write $\pi(k)$ for the number of distinct primes not exceeding $k$. From now onward, we shall always understand that $\theta_{0}$ is an effectively computable absolute constant given by Theorem 1 . Now, we combine Theorem 1 and (6) to derive the following result.

Corollary 1. Let $\varepsilon>0$ and $k \geq 27$. The equation (1) with

$$
m \geq e^{1-\theta_{0}+\varepsilon} F(k)
$$


and (5) implies that $k$ is bounded by an effectively computable number depending only on $\varepsilon$.

On the other hand, we show that Corollary 1 with $b=1$ is close to best possible in each of the assumptions (7) and (5). For this, we prove the following more general result.

TheOREM 2. Let $\varepsilon>0$. There exist effectively computable numbers $C_{3}, C_{4}$ and $C_{5}$ depending only on $\varepsilon$ such that for every pair $k, m$ with $k \geq C_{3}$ and

$$
m \leq k^{17 / 12-\varepsilon},
$$

we can find distinct integers $d_{1}, \ldots, d_{t}$ in $[1, k]$ with

(9) $t \geq \min \left(k-C_{4} \frac{k}{\log k}, k-\frac{k}{\log k}\left(1+\frac{C_{5}}{\log k}\right)\left(\log \left(\frac{m+k}{k}\right)+1+\gamma+\varepsilon\right)\right)$

and

$$
\left(m+d_{1}\right) \ldots\left(m+d_{t}\right)
$$

is a square.

If $m \leq k$, Erdös and Turk [4, p. 167] proved the assertion of Theorem 2 with (9) replaced by $t \geq k-4 k /(\log k)$. As an immediate consequence of Theorem 2, we obtain the following result.

Corollary 2. (a) Let $\varepsilon>0, k \geq 3$ and

$$
m<e^{-1-\gamma-\theta_{0}-\varepsilon} F(k) .
$$

There exists an effectively computable number $C_{6}$ depending only on $\varepsilon$ such that for $k \geq C_{6}$ there are distinct positive integers $d_{1}, \ldots, d_{t}$ not exceeding $k$ with $t$ satisfying (5) and the product is a square.

(b) Let $\varepsilon>0, k \geq 3$ and

$$
m<e^{1-\theta_{0}-\varepsilon} F(k) .
$$

The assertion of Corollary 2(a) is valid with $t$ satisfying

$$
t \geq \mu_{k}\left(\theta_{0}\right)-(2+\gamma) k /(\log k)
$$

in place of (5).

By Corollary 2(a), we observe that the assumption (7) in Corollary 1 with $b=1$ cannot be replaced by

$$
m \geq e^{-1-\gamma-\theta_{0}-\varepsilon} F(k) .
$$

Further, we see from Corollary 2(b) that we cannot relax the assumption (5) to (11) in Corollary 1 with $b=1$. 
2. Pr o of of The or em 1 . We shall choose later $\theta_{0}$, a suitable absolute positive constant. We may suppose that $k \geq c_{1}$ where $c_{1}$ is a sufficiently large effectively computable number depending only on $\theta_{0}$. Thus

$$
\varepsilon=: \frac{\theta_{0}}{\log \log k}
$$

satisfies $0<\varepsilon \leq 1 / 2$. By (1), we have

$$
m+d_{i}=A_{i} X_{i}^{2} \quad \text { for } 1 \leq i \leq t,
$$

where $A_{i}$ and $X_{i}$ are positive integers such that $P\left(A_{i}\right) \leq k$ and $A_{i}$ is square free. Further, by (4), we observe that the elements of $S_{1}=:\left\{A_{1}, \ldots, A_{t}\right\}$ are pairwise distinct. By a well-known argument of Erdős [3, Lemma 3], we find a subset $S_{2}$ of $S_{1}$ with $\left|S_{2}\right| \geq t-\pi(k)$ such that

$$
\prod_{A_{i} \in S_{2}} A_{i} \leq k^{k} \text {. }
$$

Then, we apply [10, Lemma 6] with $\eta=\varepsilon$ and

$$
g=\log \log k-\log \log \log k-\left(\theta_{0}-2\right)
$$

to conclude that there exists a subset $S_{3}$ of $S_{2}$ with

$$
\left|S_{3}\right| \geq \varepsilon k / 2
$$

and

$$
A_{i} \leq 4 e^{2} F(k) \quad \text { if } A_{i} \in S_{3} .
$$

By (13), (4) and (15), we derive that

$$
X_{i}>k^{1 / 4} \quad \text { if } A_{i} \in S_{3} .
$$

We write $S_{4}$ for the set of all $A_{i} \in S_{3}$ with $A_{i} \leq 3 k$ and let $S_{5}$ be the complement of $S_{4}$ in $S_{3}$. Now, we follow the proof of [10, Theorem 2] to derive from Erdős [3, Lemma 4] and (15) that

$$
\left|S_{5}\right| \leq \frac{12 e^{2} k}{\log \log k} .
$$

By taking $\theta_{0}>48 e^{2}$, we observe from (14), (17) and (12) that

$$
\left|S_{4}\right|>\varepsilon k / 4 \text {. }
$$

Let $C$ be as in the proof of $[9$, Theorem 2] to which we refer in this paragraph without explicit mention. We write $b_{1}, \ldots, b_{s}$ for all the integers between $k /(\log k)^{2 C}$ and $3 k$ such that every proper divisor of $b_{i}$ is less than or equal to $k /(\log k)^{2 C}$. By Brun's sieve, we derive that

$$
s \leq \frac{c_{2} k}{\log \log k}
$$


where $c_{2}$ is an effectively computable absolute constant. By taking $\theta_{0}$ sufficiently large, we derive from (18), (19) and (12) that

$$
B_{2} B_{3}\left(X_{2} X_{3}\right)^{2}=\left(B_{1} X_{1}^{2}+R\right)\left(B_{1} X_{1}^{2}+R^{\prime}\right)
$$

where $B_{1}, B_{2}, B_{3}$ and $R, R^{\prime}$ are integers of absolute values not exceeding $(\log k)^{3 C}$. For this assertion, we may permute the subscripts of $d_{1}, \ldots, d_{t}$ and this involves no loss of generality. Finally, we apply a theorem of Sprindžuk [12] (see also [9, Lemma 4]) to equation (20) to conclude from (16) that $k$ is bounded by an effectively computable absolute constant. Finally, we fix $\theta_{0}$ sufficiently large so that the arguments of the proof of Theorem 1 are valid.

3. In this section, we shall prove Theorem 2. For this, we require the following lemmas.

Lemma 1. For $x \geq 2$, we have

$$
\sum_{n \leq x} n^{-1}=\log x+\gamma+O\left(x^{-1}\right) .
$$

Pr o of. See Nagell [6, p. 276]. In particular, there is an effectively computable absolute constant $c_{3}>0$ satisfying

$$
\sum_{n \leq x} n^{-1} \leq \log x+\gamma+c_{3} x^{-1}
$$

Let $\mathcal{G}$ be a set of positive integers and denote by $\omega(\mathcal{G})$ the number of prime divisors of all the elements of $\mathcal{G}$. Then, we have

LEMma 2. There is a subset $\mathcal{G}^{\prime}$ of $\mathcal{G}$ with

$$
\left|\mathcal{G}^{\prime}\right| \geq|\mathcal{G}|-\omega(\mathcal{G})
$$

such that the product of all elements of $\mathcal{G}^{\prime}$ is a square.

Proof. See Erdős and Turk [4, Lemma 6.2].

Finally, we state the following well-known result on the number of prime factors in short intervals.

Lemma 3. Let $\varepsilon>0$. There exists $x_{0} \geq 2$ depending only on $\varepsilon$ such that for every $x \geq x_{0}$ and $h \geq x^{7 / 12+\varepsilon}$, we have

$$
\pi(x+h)-\pi(x)=\frac{h}{\log x}+O\left(\frac{h}{(\log x)^{2}}\right) .
$$

Pro of. This is due to Huxley [5]; an upper bound given by (22) is enough for our purpose. For the error term in (22), see Ramachandra [7].

Proof of Theorem 2. We put $S_{6}=\{m+1, \ldots, m+k\}$. Let

$$
\varepsilon_{1}=\left(2 c_{3}\right)^{-1} \varepsilon
$$


where $c_{3}$ is the absolute positive constant appearing in (21). We write $c_{4}, c_{5}$ and $c_{6}$ for effectively computable positive numbers depending only on $\varepsilon$. We may assume that $k \geq c_{4}$ with $c_{4}$ sufficiently large. If $m \leq k / \varepsilon_{1}$, we observe that

$$
\omega\left(S_{6}\right) \leq c_{5} k /(\log k)
$$

and we apply Lemma 2 with $\mathcal{G}=S_{6}$ to obtain the assertion of Theorem 2 . Thus, we may suppose that

$$
m>k / \varepsilon_{1} .
$$

We write $S_{7}$ for the set of all $\nu \in S_{6}$ such that $P(\nu) \leq k$. Further, we denote by $S_{8}$ the complement of $S_{7}$ in $S_{6}$. An element of $S_{8}$ is of the form $\lambda p$ where $p>k$ is a prime number and $\lambda$ is an integer satisfying $1 \leq \lambda \leq(m+k) / k$. For an integer $\lambda$ with $1 \leq \lambda \leq(m+k) / k$, we write $T_{\lambda}$ for all the elements of $S_{8}$ of the form $\lambda p$ where $p>k$ is a prime number. Further, we write

$$
t_{\lambda}=\left|T_{\lambda}\right| \quad \text { for } 1 \leq \lambda \leq(m+k) / k .
$$

Thus

$$
\left|S_{8}\right| \leq \sum_{1 \leq \lambda \leq(m+k) / k} t_{\lambda}
$$

It is clear from the definition of $T_{\lambda}$ that

$$
t_{\lambda}=\pi\left(\frac{m+k}{\lambda}\right)-\pi\left(\frac{m}{\lambda}\right) \quad \text { for } 1 \leq \lambda \leq(m+k) / k .
$$

For $1 \leq \lambda \leq(m+k) / k$, we derive from (8) that

$$
\frac{k}{\lambda}>\left(\frac{m}{\lambda}\right)^{7 / 12+\varepsilon / 2}
$$

and, by (24),

$$
\frac{m}{\lambda} \geq \frac{m k}{m+k}>\frac{k}{1+\varepsilon_{1}} .
$$

Now, we apply Lemma 3 with $x=m / \lambda, h=k / \lambda$ to derive from (26)-(28) that

$$
t_{\lambda} \leq\left(1+\frac{c_{6}}{\log k}\right) \frac{k}{\lambda(\log k)} .
$$

Next, we combine (25), (29), (21), (24) and (23) to conclude that

$$
\left|S_{8}\right| \leq G(k)
$$

where

$$
G(k)=\left(1+\frac{c_{6}}{\log k}\right) \frac{k}{\log k}\left(\log \left(\frac{m+k}{k}\right)+\gamma+\frac{\varepsilon}{2}\right) .
$$


Therefore, we obtain

$$
\left|S_{7}\right| \geq k-G(k) .
$$

Consequently, we apply Lemma 2 with $\mathcal{G}=S_{7}$ to conclude that there exists a subset $S_{9}$ of $S_{7}$ with

$$
\left|S_{9}\right| \geq k-G(k)-\pi(k)
$$

such that the product of all the elements of $S_{9}$ is a square. Finally, we observe that the right hand side of (30) is at least the right hand side of (9) with $C_{5}=c_{6}$ to complete the proof of Theorem 2 .

Remarks. (i) Without applying Lemma 3 , it is possible to obtain a slightly weaker estimate for $\left|S_{8}\right|$. By definition of $S_{8}$, we have

$$
k^{\left|S_{8}\right|} \leq \frac{(m+1) \ldots(m+k)}{k !} \leq\left(\frac{e(m+k)}{k}\right)^{k}
$$

which implies that

$$
\left|S_{8}\right| \leq \frac{k}{\log k}\left(\log \left(\frac{m+k}{k}\right)+1\right)
$$

(ii) Slight improvements of the exponent $7 / 12$ in Lemma 3 are known. Consequently, the assumption (8) in Theorem 2 can be relaxed slightly.

\section{References}

[1] A. Baker, Bounds for the solutions of the hyperelliptic equation, Proc. Cambridge Philos. Soc. 65 (1969), 439-444.

[2] P. Erdős, Note on the product of consecutive integers (I), J. London Math. Soc. 14 (1939), 194-198.

[3] -, On the product of consecutive integers III, Indag. Math. 17 (1955), 85-90.

[4] P. Erdős and J. Turk, Products of integers in short intervals, Acta Arith. 44 (1984), 147-174.

[5] M. N. Huxley, On the difference between consecutive primes, Invent. Math. 15 (1972), 164-170.

[6] T. Nagell, Introduction to Number Theory, Wiley, 1951.

[7] K. Ramachandra, Some problems of analytic number theory, Acta Arith. 31 (1976), 313-324.

[8] O. Rigge, Über ein diophantisches Problem, in: 9th Congress Math. Scand. Helsingfors, 1938, Mercator, Helsingfors, 1939, 155-160.

[9] T. N. Shorey, Perfect powers in values of certain polynomials at integer points, Math. Proc. Cambridge Philos. Soc. 99 (1986), 195-207.

[10] - Perfect powers in products of integers from a block of consecutive integers, Acta Arith. 49 (1987), 71-79.

[11] T. N. Shorey and R. Tijdeman, Perfect powers in products of terms in an arithmetical progression, Compositio Math. 75 (1990), 307-344. 
[12] V. G. Sprindžuk, Hyperelliptic diophantine equation and class numbers of ideals, Acta Arith. 30 (1976), 95-108 (in Russian).

INSTITUTE OF MATHEMATICAL SCIENCES

MADRAS 600113

TATA INSTITUTE

INDIA

OF FUNDAMENTAL RESEARCH

HOMI BHABHA ROAD

BOMBAY 400005

INDIA 